\title{
Effect of COVID-19-related Interruption of Visiting Dental Services on Pneumonia in Nursing Home Residents
}

\section{Kayoko Ito}

Niigata University Medical and Dental Hospital

\section{Yuto Ochiai}

Niigata University Graduate School of Medical and Dental Sciences

\section{Hirokazu Ashiga}

International University of Health and Welfare

\section{Hirokazu Hayashi}

Hayashi Dental Clinic

\section{Tomoko lizumi}

Health Sciences University of Hokkaido

\section{Taku Suzuki}

Minamiuonuma City Hospital

\section{Noboru Michimi}

JA Niigata Koseiren Niigata Medical Center

\section{Tetsuo Hanagata}

Hanagata Dental Clinic

\section{Sirima Kulvanich}

Niigata University Graduate School of Medical and Dental Sciences

\section{Kouta Nagoya}

Niigata University Graduate School of Medical and Dental Sciences

\section{Masahiro Watanabe}

Niigata University Graduate School of Medical and Dental Sciences Jin Magara

Niigata University Graduate School of Medical and Dental Sciences

\section{Takanori Tsujimura}

Niigata University Graduate School of Medical and Dental Sciences

Makoto Inoue ( $\boldsymbol{\nabla}$ inoue@dent.niigata-u.ac.jp )

Niigata University Graduate School of Medical and Dental Sciences 
Keywords: COVID-19, dental services, nursing home, older people, pneumonia

Posted Date: July 15th, 2021

DOI: https://doi.org/10.21203/rs.3.rs-668334/v1

License: (c) (1) This work is licensed under a Creative Commons Attribution 4.0 International License. Read Full License 


\section{Abstract}

\section{Background :}

The occurrence of pneumonia is strongly associated with oral health in older people. The study aim was to clarify the effect of the suspension of visiting dental services on the occurrence of pneumoniaduring thecoronavirus disease 2019 (COVID-19) pandemic.

\section{Methods :}

A self-administered postal questionnaire survey was conducted in 215 nursing homes in Japan. The questionnaire assessed the provision of visiting dental services and the interruption of theseservices following COVID-19emergency restrictions. Pneumonia, hospitalization, and mortality rates were examinedfor January-June 2019 and January-June 2020.

\section{Results :}

Of facilities receiving visiting dental services, $37.7 \%$ had interruptions tothoseservices. Overall, pneumonia, hospitalization, and mortality rates were lower in 2020 compared with 2019.In those facilities with nointerruptions, rates were significantly lower in 2020 compared with 2019, whereasthere was no significant difference between 2019 and 2020 in facilities with interruptions.

\section{Conclusion :}

Continuous dental services in nursing homes during the COVID-19 pandemic led to lower rates of pneumonia, hospitalization, and mortality.

\section{Backgrounds}

In Japan, $28.7 \%$ of the population was aged over 65 years in 2020 . This percentage is expected to increase annually and to reach $35 \%$ in 2040 . The number of older people who need daily nursing care has also been increasing. The Japanese Ministry of Health, Labour and Welfare has reported that 6.57 million people required daily nursing care in 2020, and this figure is expected to increase to 9.57 million over the next 20 years. Changes in population structure are associated with changes in patterns of disease. Pneumonia is now the third leading cause of death in Japan [1]. Of total cases of pneumonia, the occurrence frequency of aspiration pneumonia increases with age and is diagnosed in $70-80 \%$ of individuals aged over 70 years [2,3]. Aspiration pneumonia in older patients is caused by impairments in chewing, swallowing, and coughing functions [4]. Oral hygiene may also affect the occurrence of aspiration pneumonia. Yamanda et al. [5] found that in older patients with aspiration pneumonia, the detection rate of aerobic bacteria in sputum was highest for normal flora. Previous studies have reported poor oral hygiene and oral health status among housebound $[6,7]$ and institutionalized older people $[8,9]$. Additionally, the risk of aspiration pneumonia is greater in nursing home residents, because such patients experience many geriatric disorders [10]. 
Because of these factors, some dental professionals in Japan provide visiting dental treatment for older patients. One of the most frequently provided interventions is oral health care to maintain oral hygiene. Numerous studies show that not only salivary bacteria level, but also the occurrence frequency of high fever, pneumonia, and death in older patients is considerably reduced by professional oral health care intervention [11-14]. However, a systematic review found only low-quality evidence that professional oral health care can reduce mortality from pneumonia in nursing home residents [15].

Coronavirus disease 2019 (COVID-19) has spread rapidly worldwide and has been declared a global emergency. The total number of COVID-19 patients in Japan exceeded 44 million in March 2021 (Japanese government data). Dentistry is one of the professions with the highest exposure to COVID-19 contagion [16]. This is because patient saliva and other secretions, and materials and dental unit surfaces in contact with patients and dental personnel, can be sources of contagion for patients and personnel. Saliva and blood droplets deposited on surfaces or aerosol inhalation generated by rotating instruments and ultrasound handpieces are risks for current and future occupants of dental environments. Thus, the characteristics of the dental environment mean that the risk of cross-infection between dentists and patients may be high, and the use of disinfectants and personal protective equipment is essential [17]. Because the main routes of COVID-19 transmission are physical contact with infected patients and exposure to respiratory droplets (including those from oral and nasal cavities), dentists who providing visiting dental services have a high risk of infection through aerosol transmission [18].

Although dentists are trying to prevent the spread of infection between patients and between patients and medical personnel, many patients report that they would only visit a dentist in an emergency. Peloso et al. [19] found that patients under treatment were more worried about COVID-19 infection than patients not under treatment. Our own experience shows that many patients in nursing homes hesitate to receive care from visiting dental professionals if it is not an emergency. There are large regional differences in the spread of COVID-19 in Japan (and other countries). Patient numbers are low in rural areas in Japan, but oral health care provided by visiting dentists and dental hygienists is important to prevent pneumonia.

In this study, we collected data from residents of nursing homes in Japan. Oral health in this population is strongly associated with the occurrence of pneumonia. Many nursing homes have continued to receive visiting dental services during the pandemic, whereas others have suspended these services. The study aim was to clarify how the suspension of visiting dental services affected the occurrence of pneumonia.

\section{Materials And Methods}

Subjects

A self-administered postal questionnaire survey was conducted of 477 nursing homes for older people in Japan from July to August 2020. The facilities were as follows: 322 special nursing homes for older people and 155 geriatric health service facilities. These facilities are provided through Japan's long-term care insurance system. Special nursing homes are for individuals who are in a stable condition but 
require regular nursing care. Geriatric intermediate care facilities are for individuals receiving daily care and rehabilitation in preparation for returning home [20]. Informed consent was obtained from all nursing homes. The use of information has minimal risk to the participants and the waiver of consent will not adversely affect the rights and welfare of the participants. There were strong data security and data release protections and strict anonymization of shared data. This retrospective study was undertaken as an observational study in compliance with the Declaration of Helsinki on medical research involving human subjects and ethical approval was obtained from the Niigata University Ethics Committee (2020 0112).

\section{Questionnaires}

The questionnaires assessed the provision of visiting dental services (i.e., oral health care provided by dental personnel and regular dental examinations), the interruption of visiting dental services owing to COVID-19 emergency restrictions, and the duration of the interruption. The rates of pneumonia, hospitalization, and mortality were examined for two phases: January-June 2019 and January-June 2020. Information about issues related to oral hygiene under COVID-19 emergency restrictions, insufficient supplies for oral hygiene, countermeasures for oral hygiene, and requests to dental and administrative personnel under COVID-19 emergency restrictions was obtained using open questions.

\section{Statistical analysis}

The provision of visiting dental services, regular dental examinations, the interruption of dental services, and the duration of the interruption were analyzed separately for special nursing homes and geriatric health service facilities. The rates of pneumonia, hospitalization, and mortality by facility type and by the occurrence of interruption was assessed using the Mann-Whitney $U$ test, because the data were not normally distributed. The correlation between the duration of interruption and the rates of pneumonia, hospitalization, and mortality was analyzed using Spearman's rank correlation coefficient. All analyses were performed using the Statistical Package for the Social Sciences software version 26.0 (IBM Corp., Armonk, NY, USA). Statistical significance was set at $p<0.05$.

\section{Results}

The survey response rate was $45.1 \%$ (215 of 477 facilities); 141 special nursing homes and 74 geriatric health service facilities. Visiting dental services were provided in $85.1-87.9 \%$ facilities and regular dental examinations were performed in $29.7-39.7 \%$ of facilities (Table 1 ). The number of facilities receiving dental services, which comprised dental treatments, oral health care, and dental examinations, was 206 (95.8\%) in 2019 and 207 (96.3\%) in 2020. Of these 207 facilities, dental services were interrupted owing to COVID-19 emergency restrictions in 78 (37.7\%) facilities (Table 2). There was no difference between the percentage of special nursing homes and geriatric health service facilities in which dental services were interrupted. The duration of interruption varied from 1 to 6 months. The most frequent interruption period was 3 months (10.1\%), from March to May or from April to June. 
Table 1. Number of facilities receiving visiting dental services.

\begin{tabular}{|c|c|c|c|c|c|}
\hline & \multirow{2}{*}{$\mathrm{n}(\%)$} & \multicolumn{2}{|c|}{ Dental treatment } & \multicolumn{2}{|c|}{ Dental examination } \\
\hline & & 2019 & 2020 & 2019 & 2020 \\
\hline Total & $215(100.0 \%)$ & $189(87.9)$ & $187(87.0)$ & $79(36.7)$ & $77(35.8)$ \\
\hline Special elderly nursing home & $141(100.0 \%)$ & $124(87.9)$ & $124(87.9)$ & $56(39.7)$ & $55(39.0)$ \\
\hline Geriatric health services facility & $74(100.0 \%)$ & $65(87.8)$ & $63(85.1)$ & $23(31.1)$ & $22(29.7)$ \\
\hline
\end{tabular}

\section{Table 2. Interruption of dental servicesowingto COVID-19emergency restrictions.}

\begin{tabular}{|c|c|c|c|c|c|c|c|c|c|}
\hline & \multirow[b]{2}{*}{$\mathrm{n}(\%)$} & \multirow{2}{*}{$\begin{array}{l}\text { No of facilities } \\
\text { interrupted }\end{array}$} & \multicolumn{7}{|c|}{ Period of interruption (month) } \\
\hline & & & 1 & 2 & 3 & 4 & 5 & 6 & $\begin{array}{c}\text { Not } \\
\text { answered }\end{array}$ \\
\hline Total & $207(100.0)$ & $78(37.7)$ & $6(2.9)$ & $15(7.2)$ & $21(10.1)$ & $18(8.7)$ & $9(4.3)$ & $3(1.4)$ & $6(2.9)$ \\
\hline Special elderly nursing home & $138(100.0)$ & $53(38.4)$ & $5(3.6)$ & $9(6.5)$ & $16(11.6)$ & $12(8.7)$ & $7(5.1)$ & $2(1.4)$ & $2(1.4)$ \\
\hline Geriatric health services facility & $69(100.0)$ & $25(36.2)$ & $1(1.4)$ & $6(8.7)$ & $5(7.2)$ & $6(8.7)$ & $2(2.9)$ & $1(1.4)$ & $4(5.8)$ \\
\hline
\end{tabular}

The rates of pneumonia occurrence, hospitalization, and mortality for January-June 2019 and JanuaryJune 2020 by facility type are shown in Table 3 . The rates of pneumonia and hospitalization varied from $4.3-6.5 \%$ and from $13.7-18.1 \%$, respectively. generally, the three rates were significantly lower in 2020 compared with 2019 , except for the hospitalization rate in either facility and mortality rate in geriatric health service facilities. The mortality rate was significantly higher in special nursing homes than in geriatric health service facilities $(p<0.001)$. There was no significant correlation between duration of interruption and the rates of pneumonia, hospitalization, and mortality; the correlation coefficients were $0.075(p=0.346), 0.074(p=0.335)$, and $0.051(p=0.500)$, respectively.

Table 3. Rates of pneumonia occurrence, hospitalization, and mortality.

\begin{tabular}{|c|c|c|c|c|c|c|c|c|c|}
\hline & \multicolumn{3}{|c|}{ Rate of pnuemonia occurrence } & \multicolumn{3}{|c|}{ Rate of hospitalization } & \multicolumn{3}{|c|}{ Rate of mortality } \\
\hline & 2019 & 2020 & P-value & 2019 & 2020 & P-value & 2019 & 2020 & P-value \\
\hline Total & $5.8 \pm 5.5$ & $4.6 \pm 5.0$ & 0.001 & $16.0 \pm 11.5$ & $14.6 \pm 11.6$ & 0.038 & $9.1 \pm 6.9$ & $8.0 \pm 6.5$ & $<0.001$ \\
\hline Special elderly nursing home & $5.4 \pm 4.7$ & $4.3 \pm 4.7$ & 0.014 & $14.9 \pm 11.0$ & $13.7 \pm 11.2$ & 0.201 & $11.5 \pm 6.5$ & $10.1 \pm 6.1$ & 0.009 \\
\hline Geriatric health services facility & $6.5 \pm 6.7$ & $5.0 \pm 5.6$ & 0.015 & $18.1 \pm 12.3$ & $16.5 \pm 12.3$ & 0.097 & $4.2 \pm 4.9$ & $3.7 \pm 5.2$ & 0.105 \\
\hline P-value & 0.601 & 0.322 & - & 0.084 & 0.110 & - & $<0.001$ & $<0.001$ & - \\
\hline
\end{tabular}

We divided the pneumonia occurrence, hospitalization, and mortality rates for January-June 2019 and January-June 2020 into two groups according to the occurrence or absence of interruption of dental services (Table 4). For facilities with interrupted services, there was no significant difference in the three rates between 2019 and 2020. In contrast, for facilities with no interruptions to services, the rates were significantly lower in 2020 compared with 2019 ( $p=0.007$ for pneumonia occurrence, $p=0.015$ for 
hospitalization, and $p=0.041$ for mortality). There was no difference in the rates of pneumonia, hospitalization, and mortality between the facilities.

\section{Table 4. Effect of COVID-19-related interruptions on pneumonia occurrence,}

\section{hospitalization, and mortality.}

\begin{tabular}{|c|c|c|c|c|c|c|c|c|c|}
\hline & \multicolumn{3}{|c|}{ Rate of pnuemonia occurrence } & \multicolumn{3}{|c|}{ Rate of hospitalization } & \multicolumn{3}{|c|}{ Rate of mortality } \\
\hline & 2019 & 2020 & P-value & 2019 & 2020 & P-value & 2019 & 2020 & P-value \\
\hline Services interrupted & $5.7 \pm 5.7$ & $4.9 \pm 5.4$ & 0.057 & $15.5 \pm 11.5$ & $15.4 \pm 12.4$ & 0.603 & $9.7 \pm 7.0$ & $8.6 \pm 6.7$ & 0.066 \\
\hline Continuing services & $5.7 \pm 5.3$ & $4.3 \pm 4.7$ & 0.007 & $16.3 \pm 11.7$ & $14.2 \pm 11.3$ & 0.015 & $8.8 \pm 6.8$ & $7.8 \pm 6.5$ & 0.041 \\
\hline P-value & 0.954 & 0.507 & - & 0.674 & 0.522 & - & 0.461 & 0.415 & - \\
\hline
\end{tabular}

Issues related to oral health care during the COVID-19 pandemic are shown in Table 5. More than $40 \%$ of the facilities (42.5\%) experienced difficulties owing to postponement of dental treatment, which occurred in $30(23.2 \%)$ facilities that dental service was continued. Twenty-one facilities $(10.1 \%)$ experienced difficulty obtaining sufficient oral hygiene supplies, mainly gloves (7.7\%), masks and face shields (5.8\%), gauze (3.4\%), and hand antiseptic (2.9\%). Issues about poor oral hygiene, insufficient human resources, and increased complaints about oral hygiene occurred more in facilities with interrupted services than in facilities with uninterrupted services $(p<0.001)$.

\section{Table 5. Issues related todental service interruption owing to COVID-19emergency}

\section{restrictions.}

\begin{tabular}{lcccc}
\hline & Total & Services interrupted & Continuing services & P-value \\
\hline Total number of facilities & $207(100.0 \%)$ & $78(100.0 \%)$ & $129(100.0 \%)$ & \\
Dental treatment has been postponed & $88(42.5)$ & $58(74.3)$ & $30(23.2)$ & $<0.001$ \\
Diffficult to obtain oral care supplies & $21(10.1)$ & $6(7.7)$ & $15(11.6)$ & 0.363 \\
Poor oral hygiene increased & $18(8.7)$ & $15(19.2)$ & $3(2.3)$ & $<0.001$ \\
Insufficient human resources & $16(7.7)$ & $14(17.9)$ & $2(1.6)$ & $<0.001$ \\
Compl aints about oral cavity increased & $15(7.2)$ & $14(17.9)$ & $1(0.8)$ & $<0.001$ \\
Bad breath increased & $8(3.9)$ & $7(8.9)$ & $1(0.8)$ & 0.003 \\
Could not receive instructions for oral health care & $3(1.4)$ & $3(3.8)$ & $0(0.0)$ & 0.052 \\
\hline
\end{tabular}

As part of the oral health care countermeasures for the COVID-19 outbreak, 26 facilities adopted extra oral care measures. Of these 26 facilities, 11 experienced interruptions to dental services. Personal protective equipment was used in 23 facilities. The measures implemented by the facilities for visiting dental services included ventilation and body temperature checks of dental personnel. 
The main requests to dental and administrative personnel following the COVID-19 outbreak were as follows: continuation of dental treatment, sufficient oral care products, provision of information about the institution where the dental services are provided, an oral hygiene guideline about COVID-19 infection, oral health care instructions, and consultation with a dentist using the Internet system.

\section{Discussion}

The present survey suggested that continuous dental intervention is essential not only for oral health, but also to prevent complications such as pneumonia. Although it is important to minimize the spread of COVID-19, we should also consider the prevention of pneumonia and other diseases that can be fatal to older people.

It is surprising that the occurrence rates of pneumonia, hospitalization, and mortality significantly decreased in facilities with no interruption to dental services, and that there was no difference in 2019 and 2020 rates in facilities in which dental services were interrupted in 2020 . It may be that these complications did not increase even in facilities with interrupted dental services in 2020 because of the widespread infection countermeasures. In Japan, the first confirmed case of COVID-19 infection was recorded on January 16, 2020, and on February 3, 2020, many of the passengers and crew members of the cruise liner "Diamond Princess" were found to be infected when the ship docked in the Port of Yokohama [21]. Following the declaration of a state of emergency in April 2020 [22], personal infection control behaviors, such as mask wearing, handwashing, gargling, and social distancing [23], have been adopted. Hand hygiene is an effective infection control method in long-term care facilities for older people [24]. In addition, following government instructions to avoid unnecessary outings, family visits to such facilities have been limited. These personal infection control behaviors may suppress various infections, including pneumonia and influenza. In fact, the number of patients with influenza in Japan declined sharply from 7,285,000 in 2019 (https://www.mhlw.go.jp/content/000620714.pdf) to 140,000 in 2020 (https://www.mhlw.go.jp/content/000752481.pdf). These personal infection control behaviors should be continued even after the COVID-19 pandemic.

Therefore, we suggest that the suppression of complications was achieved by both the implementation of infectious disease countermeasures and continuation of dental services. These results emphasize the importance of dental intervention and support previous findings [4, 14].

In the present study, 78 facilities (37.7\%) stopped receiving dental services. Although we did not obtain detailed information about the interruption of dental visits, it is likely that any routine or non-urgent dental treatments were postponed. Although the interruption did not increase the prevalence of pneumonia, it is possible that the lack of dental treatment or examinations led to an increase in dental plaque, tongue coating, and dental calculus, and a worsening of caries and periodontal disease. We identified a need for continuing dental treatment, sufficient oral care products, and guidelines for oral hygiene during the COVID-19 pandemic from dental personnel and the government. There is a high risk of infection during dental treatment and oral hygiene care through aerosol transmission [18]. Additionally, dental personnel 
visiting the facilities may transmit COVID-19. There is no current guideline about the suspension of visiting dental services, and it is left to facilities and dentists to make judgments about this. There is a need for the development of a guideline for visiting dental services and oral hygiene care in geriatric facilities during pandemics of infectious diseases.

There were some study limitations. The first limitation is the possibility of sample selection bias. The survey response rate is used as an indicator of survey quality, and the validity of findings from surveys with low response rates can be questioned [25]. Sivo et al. suggest that an average mail survey response rate of $48.8 \%$ is moderately high [26]. Although our survey response rate was $45.1 \%$, it is comparable with that of a German nursing home survey (45.5\%) [27]. However, the possible of sample selection bias cannot be ruled out. The second limitation is that we did not examine the relationship between the interruption of dental services and the spread of COVID-19 in each region. Because the duration of emergency states and the number of infected people differed across regions [22], further analysis is needed. Third, the type of dental treatment and the number of patients receiving each treatment were not recorded. Fourth, the reasons for hospitalization, mortality, and the interruption of medical services were not investigated. As this survey focused on dentistry, surveys focusing on medical home visits are needed. Finally, the type of extra oral care measures that facilities adopted were not recorded.

Despite these limitations, the current results clearly demonstrate that continuation of dental services in facilities during the COVID-19 pandemic led to a decline in the rates of pneumonia, hospitalization, and mortality. The maintenance of visiting dental services is important even during an infectious disease pandemic.

\section{Conclusion}

We found a strong association of COVID-19-related interruption of dental services with the occurrence of pneumonia in dependent older people. Therefore, continuous dental services in nursing homes during the COVID-19 pandemic led to lower rates of pneumonia, hospitalization, and mortality.

\section{Declarations}

\section{Abbreviations}

Not applicable.

\section{Availability of data and materials}

The datasets used and/or analyzed during the current study are available from the corresponding author on reasonable request.

\section{Acknowledgment}


We thank Yoshimi Ohnishi, NorihiroTanaka, Akiko Yawata, and Ayako Umeharafor assistance with data collection. We also thank Diane Williams, PhD, from Edanz Group (https://en-author-

services.edanz.com/ac) for editing a draft of this manuscript.

\section{Competing interests}

The authors declare that they have no competing interests.

\section{Funding}

This research did not receive any specific grant from funding agencies in the public, commercial, ornotfor-profit sectors.

\section{Authors' contributions}

Author contributions: M.I. conceptualized the study, had full access to the data in the study and takes responsibility for the integrity of the data and the accuracy of the data analysis; H.A., H.H., T.I., T.S., N.M., T.H. were crucial in obtaining data access and data management. K.I., Y.O., S.K., S.K., K.N., M.W., J.M. and T.T. analyzed or interpreted the data. K.I., J.M., T.T. and M.I. wrote the manuscript; All authors reviewed and edited the manuscript.

\section{Ethics approval and consent to participate}

The use of information has minimal risk to the participants and the waiver of consent will not adversely affect the rights and welfare of the participants. There were strong data security and data release protections and strict anonymization of shared data.This retrospective study was undertaken as an observational study in compliance with the Declaration of Helsinki on medical research involving human subjects and ethical approval was obtained from the Niigata University Ethics Committee (2020-0112).

\section{Consent for publication}

Not applicable.

\section{References}

1. Asakura K, Azechi T, Sasano H, Matsui H, Hanaki H, Miyazaki M, Takata T, Sekine M, Takaku T, Ochiai $T$ et al: Rapid and easy detection of low-level resistance to vancomycin in methicillin-resistant Staphylococcus aureus by matrix-assisted laser desorption ionization time-of-flight mass spectrometry. PLoS One 2018, 13(3):e0194212.

2. Nihei M, Okazaki T, Ebihara S, Kobayashi M, Niu K, Gui P, Tamai T, Nukiwa T, Yamaya M, Kikuchi T et al: Chronic inflammation, lymphangiogenesis, and effect of an anti-VEGFR therapy in a mouse model and in human patients with aspiration pneumonia. The Journal of pathology 2015, 235(4):632-645. 
3. Teramoto S, Fukuchi Y, Sasaki H, Sato K, Sekizawa K, Matsuse T, Japanese Study Group on Aspiration Pulmonary D: High incidence of aspiration pneumonia in community- and hospitalacquired pneumonia in hospitalized patients: a multicenter, prospective study in Japan. J Am Geriatr Soc 2008, 56(3):577-579.

4. Yamaya $\mathrm{M}$, Yanai $\mathrm{M}$, Ohrui $\mathrm{T}$, Arai $\mathrm{H}$, Sasaki $\mathrm{H}$ : Interventions to prevent pneumonia among older adults. J Am Geriatr Soc 2001, 49(1):85-90.

5. Yamanda S, Ebihara S, Ebihara T, Yamasaki M, Arai H, Kohzuki M: Bacteriology of aspiration pneumonia due to delayed triggering of the swallowing reflex in elderly patients. The Journal of hospital infection 2010, 74(4):399-401.

6. Morishita M, Mori S, Yamagami S, Mizutani M: Effect of carbonated beverages on pharyngeal swallowing in young individuals and elderly inpatients. Dysphagia 2014, 29(2):213-222.

7. Strayer MS: Perceived barriers to oral health care among the homebound. Special care in dentistry : official publication of the American Association of Hospital Dentists, the Academy of Dentistry for the Handicapped, and the American Society for Geriatric Dentistry 1995, 15(3):113-118.

8. Knabe C, Kram P: Dental care for institutionalized geriatric patients in Germany. J Oral Rehabil 1997, 24(12):909-912.

9. Miyazaki H, Shirahama R, Ohtani I, Shimada N, Takehara T: Oral health conditions and denture treatment needs in institutionalized elderly people in Japan. Community Dent Oral Epidemiol 1992, 20(5):297-301.

10. Muder RR: Pneumonia in residents of long-term care facilities: epidemiology, etiology, management, and prevention. Am J Med 1998, 105(4):319-330.

11. Adachi M, Ishihara K, Abe S, Okuda K: Professional oral health care by dental hygienists reduced respiratory infections in elderly persons requiring nursing care. International journal of dental hygiene 2007, 5(2):69-74.

12. Ishikawa A, Yoneyama T, Hirota K, Miyake Y, Miyatake K: Professional oral health care reduces the number of oropharyngeal bacteria. J Dent Res 2008, 87(6):594-598.

13. Scannapieco FA, Shay K: Oral health disparities in older adults: oral bacteria, inflammation, and aspiration pneumonia. Dental clinics of North America 2014, 58(4):771-782.

14. Yoneyama T, Yoshida M, Ohrui T, Mukaiyama H, Okamoto H, Hoshiba K, Ihara S, Yanagisawa S, Ariumi S, Morita T et al: Oral care reduces pneumonia in older patients in nursing homes. J Am Geriatr Soc 2002, 50(3):430-433.

15. Liu C, Cao Y, Lin J, Ng L, Needleman I, Walsh T, Li C: Oral care measures for preventing nursing homeacquired pneumonia. Cochrane Database Syst Rev 2018, 9:CD012416.

16. Villani FA, Aiuto R, Paglia L, Re D: COVID-19 and Dentistry: Prevention in Dental Practice, a Literature Review. International journal of environmental research and public health 2020, 17(12).

17. Luzzi V, lerardo G, Bossu M, Polimeni A: Paediatric Oral Health during and after the COVID-19 Pandemic. Int J Paediatr Dent 2021, 31(1):20-26. 
18. Kimura Y, Ueha R, Furukawa T, Oshima F, Fujitani J, Nakajima J, Kaneoka A, Aoyama H, Fujimoto Y, Umezaki T: Society of swallowing and dysphagia of Japan: Position statement on dysphagia management during the COVID-19 outbreak. Auris, nasus, larynx 2020, 47(5):715-726.

19. Peloso RM, Pini NIP, Sundfeld Neto D, Mori AA, Oliveira RCG, Valarelli FP, Freitas KMS: How does the quarantine resulting from COVID-19 impact dental appointments and patient anxiety levels? Brazilian oral research 2020, 34:e84.

20. Morita K, Ono S, Ishimaru M, Matsui H, Naruse T, Yasunaga H: Factors Affecting Discharge to Home of Geriatric Intermediate Care Facility Residents in Japan. J Am Geriatr Soc 2018, 66(4):728-734.

21. Watanabe M: The COVID-19 Pandemic in Japan. Surg Today 2020, 50(8):787-793.

22. Karako K, Song P, Chen Y, Tang W, Kokudo N: Overview of the characteristics of and responses to the three waves of COVID-19 in Japan during 2020-2021. Biosci Trends 2021, 15(1):1-8.

23. Nishi M: Jishuku, social distancing and care in the time of COVID-19 in Japan. Soc Anthropol 2020.

24. Hocine MN, Temime L: Impact of hand hygiene on the infectious risk in nursing home residents: $A$ systematic review. Am J Infect Control 2015, 43(9):e47-52.

25. Harrison S, Alderdice F, Quigley MA: External validity of prevalence estimates from the national maternity surveys in England: The impact of response rate. PLoS One 2020, 15(11):e0242815.

26. Sivo SA, Saunders C, Chang Q, Jiang JJ: How Low Should You Go? Low Response Rates and the Validity of Inference in IS Questionnaire Research. Journal of the Association for Information Systems 2006, 7(6):351-414.

27. Strautmann A, Allers K, Fassmer AM, Hoffmann F: Nursing home staff's perspective on end-of-life care of German nursing home residents: a cross-sectional survey. BMC Palliat Care 2020, 19(1):2. 